\title{
Surgical management of isolated retroperitoneal Castleman's disease: A case report
}

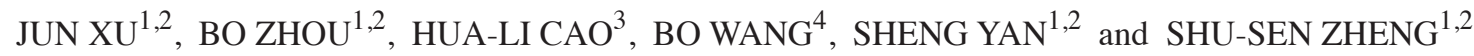 \\ ${ }^{1}$ Department of Hepatobiliary and Pancreatic Surgery, First Affiliated Hospital, Zhejiang University School of Medicine; \\ ${ }^{2}$ Key Laboratory of Combined Multi-Organ Transplantation, Ministry of Public Health; ${ }^{3}$ Department of \\ Dermatology, Second Affiliated Hospital; ${ }^{4}$ Department of Pathology, First Affiliated Hospital, \\ Zhejiang University School of Medicine, Hangzhou, Zhejiang 310003, P.R. China
}

Received March 11, 2015; Accepted December 23, 2015

DOI: $10.3892 / \mathrm{ol} .2016 .4177$

\begin{abstract}
Castleman's disease (CD) is an uncommon, poorly understood lymphoproliferative disease. Retroperitoneal forms may present as either a unicentric or multicentric disease. The present study reports the case of a 36-year-old man who was referred to the First Affiliated Hospital, School of Medicine, Zhejiang University (Hangzhou, China), for a detailed examination of an abdominal mass. The abdominal ultrasound and computed tomography scans revealed a solid mass localized in the region between segment 1 of the liver and the pancreas. An endosonography-guided fine-needle aspiration biopsy revealed chronic inflammation and lymphadenosis. The present study reports a rare case, in which the patient was treated with an exploratory laparotomy and resection. The retroperitoneal mass was pathologically diagnosed as CD of the hyaline vascular type. The patient was closely followed-up for 11 months and is presently free of disease. In conclusion, the possibility of unicentric CD should be considered when facing a solid hypervascular retroperitoneal mass. A complete surgical resection may successfully treat the disease without an unnecessarily extensive resection for the unicentric type.
\end{abstract}

\section{Introduction}

Castleman's disease (CD) is a relatively rare lymphoproliferative disease, characterized by enlarged hyperplastic lymph node(s) (1). The disease was initially described by

Correspondence to: Professor Shu-Sen Zheng, Department of Hepatobiliary and Pancreatic Surgery, First Affiliated Hospital, Zhejiang University School of Medicine, 79 Qingchun Road, Hangzhou, Zhejiang 310003, P.R. China

E-mail: shusenzheng@zju.edu.cn

Abbreviations: CD, Castleman's disease; HHV-8, human herpes virus 8 infection; HIV, human immunodeficiency virus; CT, computed tomography

Key words: Castleman's disease, hyaline type, laparotomy
Castleman and Towne in 1954 (2). The disease most commonly develops in the mediastinum, and the cervical region is the second most common location. Additional common nonmediastinal sites are the pelvic cavity, axilla and retroperitoneum $(3,4)$. Clinically, CD may manifest as localized disease (unicentric) or widespread disease (multicentric) (3). The unicentric type of CD typically presents as an asymptomatic singular enlarged lymph node. By contrast, the multicentric type of CD is associated with systemic symptoms (3). CD is also classified according to histopathological type into hyaline vascular, plasma cell or mixed cell type (5). The hyaline vascular type accounts for $90 \%$ of all CD cases, and is most common in 30-40-year-old females. The plasma cell type accounts for $\sim 10 \%$ of cases, and typically occurs in patients of 50-60 years of age (6). At present there is no standard protocol for predicting the prognosis and effectively managing CD. The present study reports a rare case of unicentric CD, which presented as a retroperitoneal mass and was located in a peripancreatic location, in an asymptomatic 36-year-old male patient.

\section{Case report}

An asymptomatic 36-year-old man with an unremarkable past medical history received a routine physical examination in Yiqiao Town Central Hospital (Hangzhou, China) in March 2014, and was indicated to have an abdominal mass upon ultrasonography and computed tomography (CT) scans. The patient was then referred to the First Affiliated Hospital, School of Medicine, Zhejiang University (Hangzhou, China).

A physical examination at the First Affiliated Hospital did not reveal any abnormal results, and the patient's full blood count and biochemical profile were normal. The result of the human immunodeficiency virus (HIV) screening test was negative.

An abdominal ultrasound (MyLab ${ }^{\mathrm{TM}}$ Twice; Esaote Europe $\mathrm{BV}$, Cambridge, UK) revealed a $67 \times 55-\mathrm{mm}$ hypoechoic mass in the region between the first liver segment and the pancreas, which possessed calcification on the inside. The abdominal contrast-enhanced CT scan (Brilliance iCT; Philips Healthcare, Andover, MA, USA) reveled a 70x72-mm mass on the border of the caudate lobe. The non-enhanced phase revealed patchy calcification inside the mass (Fig. 1A), and evident 


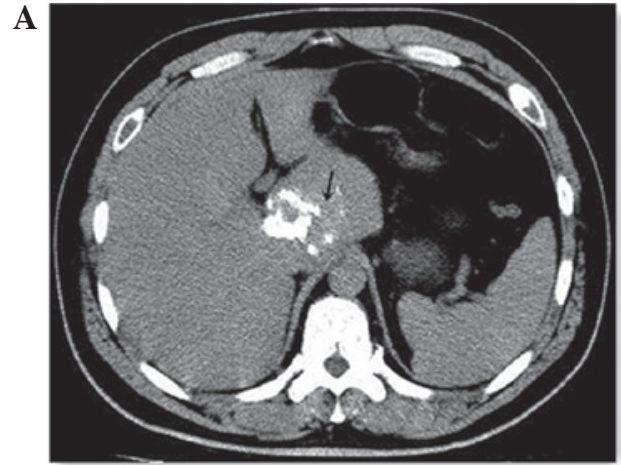

B

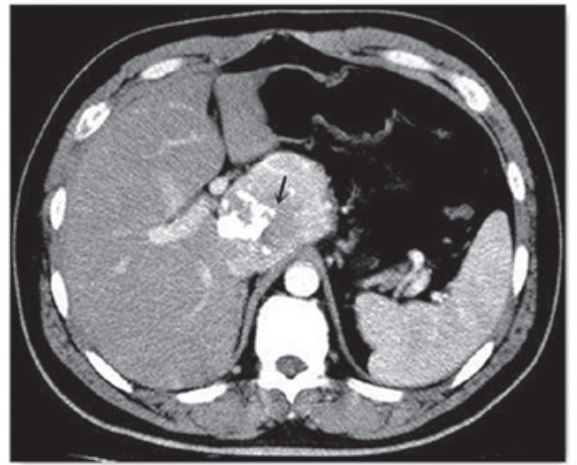

$\mathbf{C}$

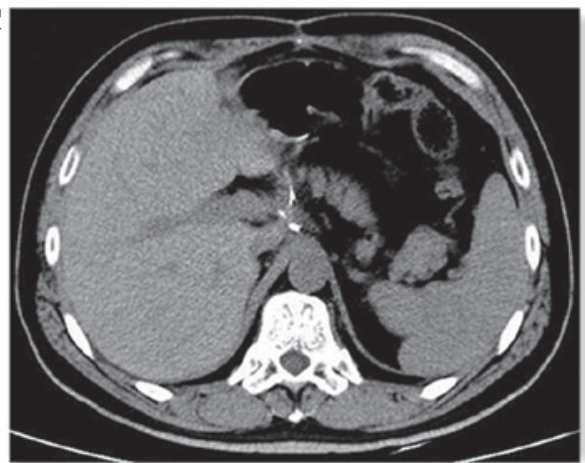

D

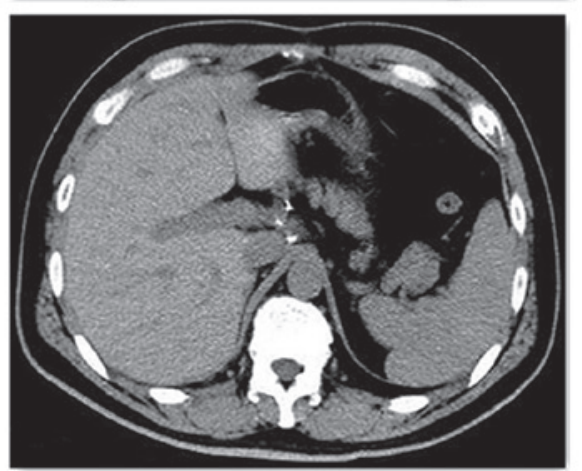

Figure 1 Pre-operative and post-operative computed tomography showing the retroperitoneal tumor (arrow) in the (A) non-enhanced phase, (B) the arterial phase, $(\mathrm{C})$ at 1 month post-surgery and (D) at 5 months post-surgery.

contrast enhancement was observed in the mass during the arterial phase (Fig. 1B). The CT scan also revealed possible infiltration into the head and neck of the pancreas, indicating a solid pseudopapillary tumor of the pancreas.

The endosonography revealed a $71 \times 51-\mathrm{mm}$ retroperitoneal mass infiltrating into the pancreas hook, indicating neuroendocrine carcinoma originating from the pancreas. An endosonography-guided fine-needle aspiration biopsy revealed chronic inflammation and lymphadenosis (Fig. 2).

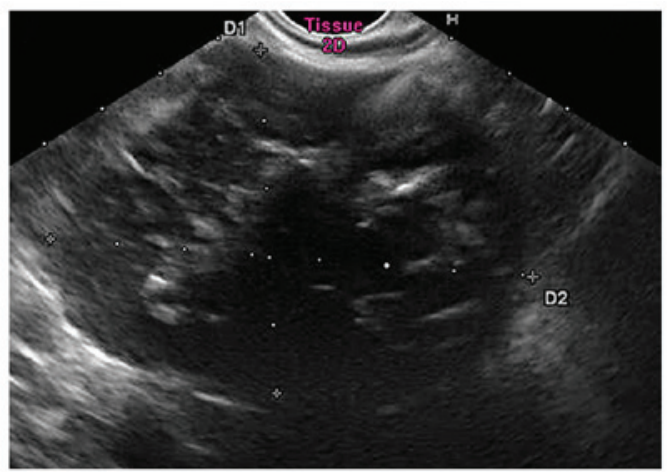

Figure 2. Endosonography. A retroperitoneal mass was detected infiltrating the pancreas hook.

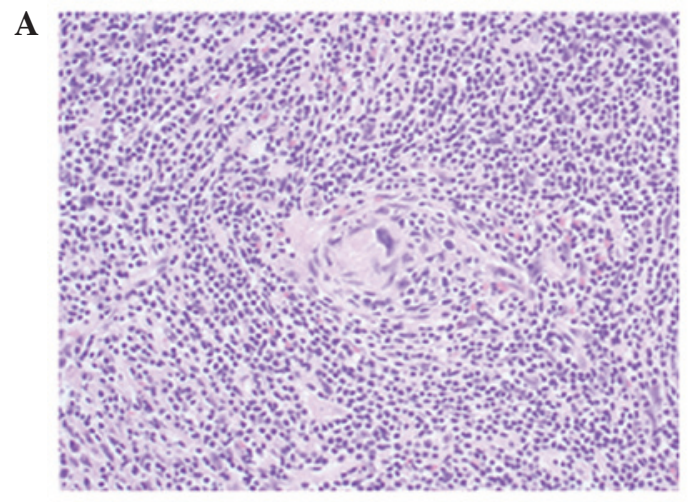

B

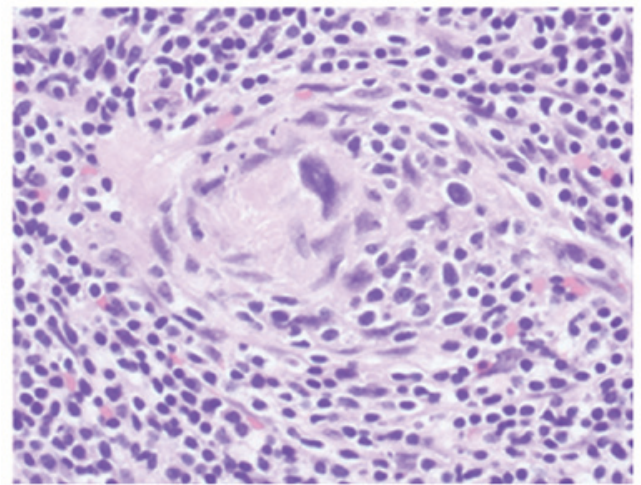

Figure 3. Histopathological presentation of the patient's lesions. Hematoxylin and eosin staining revealing lymphoid tissue with angiofolicular hyperplasia, as a hyaline vascular type of Castleman's disease. (A) Original magnification, x200; (B) original magnification, $x 400$.

On the basis of these test results, the patient underwent an exploratory laparotomy. This procedure revealed a well-defined mass densely adherent to the celiac trunk, inferior vena cava and superior border of the pancreas. The mass was excised from the adjacent organs successfully, without any complications.

The excised mass was sectioned as follows: The paraffin-embedded tissue was sectioned continuously into slices of $4 \mu \mathrm{m}$ thickness using a Leica CM1850 freezing microtome (Leica Biosystems, Wetzlar, Germany) and subsequently transferred onto the surface of prepared glass slides (Thermo Fisher Scientific, Inc., Waltham, MA, USA).

The histological examination revealed a mass consisting of lymphoid tissue with a large amount of vascular invasion, 
and laminated mantle zones with concentric rings of small lymphocytes surrounding small atrophic germinal centers. A hyalinized interstitium was observed between follicles (Fig. 3).

Immunohistochemistry for the biomarkers was performed manually. Briefly, antigen retrieval was performed using citrate buffer (pH 6.0; Sangon Biotech Co., Ltd., Shanghai, China). Sections were subsequently incubated with the following antibodies: Mouse monoclonal anti-human cluster of differentiation 20 (clone 7D1; dilution, 1:200; catalog no., NCL-CD20-7D1; Leica Biosystems), mouse monoclonal anti-human cluster of differentiation 3 (clone LN10; dilution, 1:100; catalog no., NCL-L-CD3-565; Leica Biosystems), mouse monoclonal anti-human cluster of differentiation 21 (clone 2G9; dilution, 1:60; catalog no., NCL-CD21-2G9; Leica Biosystems) and rabbit anti-human immunoglobulin $\mathrm{G}$ cluster of differentiation 23 (clone RM104; dilution, 1:100; catalog no., RM-9123-S0; Thermo Fisher Scientific, Inc.), in a humidified chamber for $1 \mathrm{~h}$ at $37^{\circ} \mathrm{C}$. Sections were then incubated with horseradish peroxidase-conjugated goat anti-mouse secondary antibody (part of the EnVision+ System-HRP kit; ready to use; catalog no., K4007; Dako, Glostrup, Denmark). Following incubation, 3,3'-diaminobenzidine (part of the EnVision+ System-HRP kit) was applied for $\sim 2 \mathrm{~min}$. Slides were subsequently stained with hematoxylin (Sangon Biotech Co., Ltd., Shanghai, China). A negative control was designed by using phosphate-buffered saline (Sangon Biotech Co., Ltd., Shanghai, China) rather than primary antibody. The immunohistochemical analysis revealed the expression of cluster of differentiation 3, 20, 21 and 23 subsequent to staining. Due to these findings, the patient was diagnosed with CD of the hyaline vascular type. The patient was followed up and is currently free of disease 20 months subsequent to resection (Fig. 1C and D).

\section{Discussion}

CD exhibits a low incidence rate worldwide. Histologically, there are 3 types of CD: The hyaline vascular type, the plasma cell type and the mixed type (5,7). Approximately $90 \%$ of cases are the hyaline vascular type (8). Clinically, according to the range of lymph nodes involved, CD may also be divided into the unicentric and multicentric types (9). The hyaline vascular and plasma cell types have often been recognized as being associated with the unicentric and multicentric presentations, respectively $(10,11)$.

The clinical presentations of $\mathrm{CD}$ vary greatly between the unicentric and multicentric types. Patients with unicentric disease are usually asymptomatic or may present with lymph node swelling. Cases of the unicentric type usually have a good prognosis and are treated with surgical excision (3). By contrast, constitutional symptoms, including fatigue, fever, sweat, weight loss, arthralgia, hepatomegaly, splenomegaly and systemic lymph node swelling, are often observed in patients with multicentric disease $(5,12)$. The presentations of the multicentric type are occasionally accompanied by symptoms of POEMS, including polyneuritis, organomegaly, endocrinopathy, M protein and skin changes $(13,14)$. Patients with the multicentric type tend to possess an unfavorable prognosis, and a number of cases eventually develop lymphomas (15-17).

The etiology of CD remains unclear and has been described in association with a reactive lymphoid hyperplasia initiated by viral infections or a developmental growth disturbance of the lymphoid tissue $(1,18)$. Certain studies have indicated that $\mathrm{CD}$ is associated with human herpes virus 8 (HHV-8) and HIV infection $(19,20)$. These viruses may lead to the oversecretion of inflammatory mediators, particularly interleukin-6 $(21,22)$, which results in the induction of a hyperplasic reaction of the lymphoid system.

The initial challenge in retroperitoneal $C D$ remains in the establishment of a diagnosis. However, CD is often overlooked as a possible diagnosis due to its low incidence. Imaging tests have been shown to aid diagnosis. The possibility of CD should be considered following the identification of a homogeneous vascular mass with contrast kinetics that follow the surrounding large arteries along a lymph node chain (23). CD is ultimately diagnosed by histology, which requires either a resection or biopsy of the lesion in order to be definitive. A number of previous cases had diagnosed abdominal unicentric $\mathrm{CD}$ following post-surgical histological examination. The preoperative diagnoses in these cases were pheochromocytoma (6), malignant tumor (24) and unknown mass $(25,26)$. The majority of $\mathrm{CD}$ cases receive immediate resection, however, Bo et al (27) performed an initial exploratory laparotomy prior to excision. Attention to the patient's history and imaging results may assist with the diagnosis of CD (23). In the present study, a fine-needle aspiration biopsy was not a useful diagnostic tool for $\mathrm{CD}$, which is similar to the findings of other studies (28-30). This issue may be attributed to the fact that the histological diagnosis of CD is based on cell architecture (30). Therefore, similar to previous cases $(3,8,23)$, a surgical resection was used for the diagnosis and treatment of the present patient. To the best of our knowledge, this strategy was rational for the present case.

In conclusion, the rare case of a patient with a retroperitoneal unicentric $\mathrm{CD}$ of hyaline vascular type was encountered during a routine physical examination. A well-defined mass densely adherent to the celiac trunk, inferior vena cava and superior border of the pancreas was identified. Overall, CD has a good prognosis following the surgical removal of the lesion. The possible diagnosis of unicentric CD should be considered in the presence of a solid hypervascular retroperitoneal mass. In cases where a unicentric type of CD is suspected, a complete surgical resection may be used to successfully treat the patient without an unnecessarily extensive resection.

\section{References}

1. El-Osta HE and Kurzrock R: Castleman's disease: From basic mechanisms to molecular therapeutics. Oncologist 16: 497-511, 2011.

2. Castleman B and Towne VW: Case records of the Massachusetts General Hospital; weekly clinicopathological exercises; founded by Richard C. Cabot. N Engl J Med 251: 396-400, 1954.

3. Bucher P, Chassot G, Zufferey G, Ris F, Huber O and Morel P: Surgical management of abdominal and retroperitoneal Castleman's disease. World J Surg Oncol 3: 33, 2005.

4. Fu L, Wang XL, Babu SR, Zhang Y, Su AP, Wang ZL, Hu T and Tian BL: Pancreatic Castleman's disease: Studies of three cases and a cumulative review of the literature. Indian J Surg 75: 34-38, 2013.

5. Keller AR, Hochholzer L and Castleman B: Hyaline-vascular and plasma-cell types of giant lymph node hyperplasia of the mediastinum and other locations. Cancer 29: 670-683, 1972.

6. Zhang J, Song N, Liu B, Hua L, Wang Z, Yin C and Zhang W: A case report of unusual retroperitoneal Castleman's disease in an old woman. Urol Int 89: 369-372, 2012. 
7. Flendrig JA and Schillings PHM: Benign giant lymphoma: The clinical signs and symptoms. Folia Med Neerl 12: 119-120, 1969.

8. Seco JL, Velasco F, Manuel JS, Serrano SR, Tomas L and Velasco A: Retroperitoneal Castleman's disease. Surgery 112 850-855, 1992

9. Gaba AR, Stein RS, Sweet DL and Variakojis D: Multicentric giant lymph node hyperplasia. Am J Clin Pathol 69: 86-90, 1978

10. Frizzera G, Banks PM, Massarelli G and Rosai J: A systemic lymphoproliferative disorder with morphologic features of Castleman's disease. Pathological findings in 15 patients. Am J Surg Pathol 7: 211-231, 1983.

11. Talat N and Schulte KM: Castleman's disease: Systematic analysis of 416 patients from the literature. Oncologist 16 1316-1324, 2011

12. Shahidi H, Myers JL and Kvale PA: Castleman's disease. Mayo Clin Proc 70: 969-977, 1995.

13. Gracia-Ramos AE, Cruz-Domínguez MdelP and Vera-Lastra OL: Multicentric hyaline vascular Castleman's disease. A POEMS type variant. Rev Med Inst Mex Seguro Soc 51: 464-467, 2013 (In Spanish).

14. Andhavarapu S and Jiang L: POEMS syndrome and Castleman disease. Blood 122: 159, 2013.

15. Franco V: Report of a case of localized Castleman's disease with progression to malignant lymphoma. Am J Clin Pathol 100: 707-708, 1993

16. Park J, Lee JE, Kim M, Lim J, Kim Y, Han K, Park G, Jung YH, Roh SY and Hong YS: Discordant lymphocyte-depleted classical Hodgkin's and peripheral T-cell lymphoma arising in a patient 11 years after diagnosis of multicentric Castleman's disease. Int J Hematol 98: 114-121, 2013.

17. Rao S, Ramesh A, Rajkumar A, Arcot R and Kuruvilla S: Persistent or recurrent Castleman's disease - Look out for a lurking lymphoma! Indian J Med Paediatr Oncol 32: 162-164, 2011.

18. Ozkan H, Tolunay S, Gözü O and Ozer ZG: Giant lymphoid hamartoma of mediastinum (Castleman's disease). Thorac Cardiovasc Surg 38: 321-323, 1990.

19. Dossier A, Meignin V, Fieschi C, Boutboul D, Oksenhendler E and Galicier L: Human herpes virus 8-related Castleman disease in the absence of HIV infection. Clin Infect Dis 56: 833-842, 2013.
20. Oksenhendler E: HIV-associated multicentric Castleman disease. Curr Opin HIV AIDS 4: 16-21, 2009.

21. Yoshizaki K, Matsuda T, Nishimoto N, Kuritani T, Taeho L, Aozasa K, Nakahata T, Kawai H, Tagoh H, Komori T, et al: Pathogenic significance of interleukin-6 (IL-6/BSF-2) in Castleman's disease. Blood 74: 1360-1367, 1989.

22. Suthaus J, Stuhlmann-Laeisz C, Tompkins VS, Rosean TR, Klapper W, Tosato G, Janz S, Scheller J and Rose-John S: HHV-8-encoded viral IL-6 collaborates with mouse IL-6 in the development of multicentric Castleman disease in mice. Blood 119: 5173-5181, 2012.

23. Poole PS, Chang EY and Santillan CS: Case 172: Retroperitoneal Castleman disease (hyaline vascular type). Radiology 260: 601-605, 2011.

24. Miyoshi H, Mimura S, Nomura T, Tani J, Morishita A, Kobara H, Mori H, Yoneyama H, Deguchi A, Himoto T, et al: A rare case of hyaline-type Castleman disease in the liver. World J Hepatol 5: 404-408, 2013

25. Otto M, Wieprzowski L, Dzwonkowski J and Ziarkiewicz-Wróblewska B.: Castleman's disease - an unusual indication for laparoscopic adrenalectomy. Wideochir Inne Tech Maloinwazyjne 7: 50-54, 2012.

26. Kim MS, Ju JK and Kim Y: Surgical management of unicentric castleman's disease in the abdomen. Ann Coloproctol 30: 97-100, 2014.

27. Bo P, Junhua Z, Qiruo G and Hong L: A case report of retroperitoneal Castleman disease. Can Urol Assoc J 3: E14-E16, 2009.

28. Park JB, Hwang JH, Kim H, Choe HS, Kim YK, Kim HB and Bang SM: Castleman disease presenting with jaundice: A case with the multicentric hyaline vascular variant. Korean J Intern Med 22: 113-117, 2007.

29. RheeKH,LeeSS andHuhJR:Endoscopicultrasonography-guided trucut biopsy for the preoperative diagnosis of peripancreatic Castleman's disease: A case report. World J Gastroenterol 14: 2115-2117, 2008.

30. Cecka F, Ferko A, Jon B, Subrt Z, Kasparova P and Repak R: Pancreatic Castleman disease treated with laparoscopic distal pancreatectomy. Hepatobiliary Pancreat Dis Int 12: 332-334, 2013. 